\title{
Análisis de las estrategias de marketing relacional en instituciones de educación superior de Colombia y España
}

\section{Analysis of relational marketing strategies in higher education institutions in Colombia and Spain}

\begin{abstract}
Mg. Ledy Gómez-Bayona es profesora e investigadora de la Universidad de San Buenaventura-Medellín (Colombia) (ledy.gomez@usbmed.edu.co) (https://orcid.org/0000-0003-4122-0344)
\end{abstract}

Dr. Juan Pablo Arrubla-Zapata es profesor e investigador de la Universidad de Medellín (Colombia) (jarrubla@udem.edu.co) (https://orcid.org/0000-0002-6240-3564)

Julián Aristizábal Valencia es integrante del semillero Xplomarketing y estudiante de Negocios Internacionales de la Universidad de San Buenaventura-Medellín (Colombia) (https://orcid.org/0000-0003-4631-2715)

María José Restrepo-Rojas es integrante del semillero Xplomarketing y estudiante de Contaduría Pública de la Universidad de San Buenaventura-Medellín (Colombia) (https://orcid.org/0000-0003-4627-910X)

\section{Resumen}

Las estrategias de marketing en las universidades han permitido una mejor comprensión de las audiencias y la clasificación de acciones que le generen valor a la marca universitaria. Es por esto que el presente proyecto investigativo pretende identificar la importancia de las estrategias de marketing relacional en el sector educativo, especialmente en las principales universidades de Colombia y España para conocer de qué manera implementan acciones mercadológicas que generan valor. La metodología utilizada para lograr este objetivo fue a partir de una revisión de literatura, donde se analizó la evolución y los modelos de marketing aplicados en educación; de igual manera, se examinaron las principales variables de marketing con enfoque relacional y se hicieron comparativos entre los países mencionados para encontrar la diferencia en las estrategias que cada uno implementa. Se concluye que, tanto en Colombia como en España, se empiezan a planear estrategias de marketing con enfoque relacional que generan valor a la gestión académica y administrativa; así mismo se evidencia que las principales variables — creación de valor, satisfacción y confianza— para las diferentes acciones implementadas en las universidades de los países analizados son pilares fundamentales para la incorporación de un buen marketing relacional en educación.

\begin{abstract}
Marketing strategies in universities have allowed a better understanding of the audiences and the classification of actions that generate value to the university brand. That is why this research project aims to identify the importance of relationship marketing strategies in the education sector, especially in the main universities in Colombia and Spain to know how they implement marketing actions that generate value. The methodology used to achieve this objective was based on a review of literature, where the evolution and models of marketing applied in education were analyzed; likewise, the main variables of marketing with a relational approach were examined and comparisons were made between the countries mentioned to find the difference in the strategies that each one implements. It is concluded that, both in Colombia and in Spain, marketing strategies with a relational focus are beginning to be planned that generate value to academic and administrative management; likewise, it is evident that the main variables - creation of value, satisfaction and confidence - for the different actions implemented in the universities of the countries analyzed are fundamental pillars for the incorporation of good relational marketing in education.
\end{abstract}

\section{Palabras clave I keywords}

Marketing, marketing relacional, estrategia, gestión, universidades, estudiante, docente, servicio. Marketing, relationship marketing, strategy, management, higher education, student, teacher, service.

Cómo citar: Gómez-Bayona, L., Arrubla-Zapata, J.P., Aristizábal-Valencia, J., y Restrepo-Rojas, M.J. (2018). Análisis de las estrategias de marketing relacional en instituciones de educación superior de Colombia y España. Retos Revista de Ciencias de la Administración y Economía, 10(20), pp. 343-359. https://doi.org/10.17163/ret.n20.2020.09 


\section{Introducción}

Comprender el marketing en la actualidad resulta un importante reto para los directivos de las organizaciones, anteriormente esta disciplina se utilizaba en empresas que tenían como enfoque la comercialización de tangibles, pero en la actualidad casi todas las empresas requieren incorporar planes estratégicos de marketing para potencializar resultados. Al analizar esta disciplina mercadológica en las universidades, se evidencia la incorporación de acciones innovadoras que permiten posicionar y visibilizar la marca universitaria (Peralta-Silva \& Linares-Cazola, 2013). El enfoque del marketing en la gestión administrativa ha evolucionado hacia una comunicación mucho más cercana con los diferentes públicos de interés, esto con el fin de construir un relacionamiento más duradero con ellos (Hennig-Thurau, Langer, \& Hansen 2001) y construir un compromiso de contenido y concepto de marca pertinente a partir de las diferentes plataformas digitales con las que se cuentan actualmente (Peruta \& Shields 2018). La formación de individuos es un aspecto de gran relevancia e impacto en el desarrollo global, esto por lo cambios significativos que ellos pueden impulsar en la sociedad. La educación constituye una escalera hacia el conocimiento que permite ir avanzando, tanto personal como profesionalmente, en esta medida es de gran importancia en el crecimiento de una ciudad, de un país y en general del mundo (CeballosLozano et al., 2012). Ahora bien, la globalización de los servicios educativos, la crisis de financiamiento y la creciente competencia que proviene del sector privado han obligado a las instituciones de educación superior a considerar el fortalecimiento de las relaciones con sus grupos de interés como la clave para el éxito futuro (Fernández et al., 2007), para ofrecer servicios de calidad y que generen valor en cada acción para consolidarse en el mercado (Sakthivel \& Raju, 2006), siendo efectivos en el servicio y dando respuesta con prontitud (Cownie, 2019).

Es necesario analizar de qué manera el marketing relacional ha permeado la gestión de las universidades para construir portafolios de calidad y en articulación con los grupos de interés (Arnett et al., 2003; Webster \& Hammond, 2008), para ello, se reconoce que el concepto de marketing relacional ha cobrado especial importancia en el ámbito de los servicios, ha considerado variables tales como la confianza, el valor, el compromiso o la lealtad (Bryce, 2007), en donde todas estas son necesarias para el desarrollo, mantenimiento y fortalecimiento de relaciones a largo plazo con los diferentes grupos de interés (Trullas et al., 2018), sin embargo, la interrelación entre los estudios en el ámbito de los servicios educativos y la línea de investigación en marketing de servicios es todavía muy escasa (Hennig-Thurau et al., 2001). La siguiente investigación tiene el objetivo de analizar el marketing relacional en el contexto educativo, como una disciplina especializada en el estudio del servicio, la calidad y la satisfacción, que a su vez se hace necesaria en toda organización para entender el entorno competitivo, enfocándose en demostrar cómo el marketing relacional es una herramienta importante para la realización de nuevas estrategias que permitan a las instituciones universitarias tener un mayor fortalecimiento en las relaciones con sus grupos de interés a los cuales van dirigidos su servicios educativos. De la misma manera, viendo este enfoque del marketing relacional en las universidades de España y de Colombia, observando también las estrategias que estas implementan para el relacionamiento constante en el sector educativo. Por todo esto, es oportuno dar repuesta al 
siguiente interrogante: ¿Cuáles son las estrategias que implementan las instituciones de educación superior en cuanto al marketing relacional, para un mejor relacionamiento con los grupos de interés? Para lo cual, se llevó a cabo un desarrollo teórico en la evolución del marketing en educación, del marketing relacional y la importancia de la gestión educativa, posterior se da a conocer el método de revisión de literatura utilizado y los resultados alcanzados, junto con las conclusiones de la investigación.

\subsection{Evolución del marketing desde el año 2000 hasta la actualidad}

A través de los años el marketing ha tenido una constate evolución, donde ha tomado diferentes perspectivas dependiendo de la necesidad del mercado (Oplatka \& Lapidot 2018), por esto han existido diferentes tendencias de marketing como lo son: el marketing educativo, relacional, transaccional y tradicional (James et al., 2019). El marketing debe entenderse más como un proceso interactivo de gestión que como una función, ya que la gestión orientada al mercado se construye mejor sobre la relación que sobre la transacción (Barroso \& Martín, 1999), y por lo tanto se observa que a partir del desarrollo de los individuos, el comportamiento de los mercados se afecta y las organizaciones implementan acciones que generen cultura de servicio, con lo que se concluye que el marketing se encuentra en todo, no solamente en lo formal, sino también en lo informal (Córdoba-López, 2009). Durante los últimos 60 años, el marketing ha pasado de estar enfocado únicamente en el producto, a hacerlo en el cliente, a lo que anteriormente se le denominaba marketing 1.0. Más tarde, cuando las compañías se percataron que el cliente es la fuente vital y parte fundamental de sus compañías, se inclinó por hacer de esta nueva fuerza el centro de sus estrategias y decisiones, creándose entonces así el marketing 2.0. Actualmente, el marketing ha tenido una evolución más en la nueva dinámica del mercado, en donde se puede observar cómo las empresas amplían su foco de negocio hacia asuntos más humanistas, donde la rentabilidad debe estar mucho más equilibrada con la responsabilidad corporativa (Gómez \& Uribe, 2016), por ende, se da la creación a lo que actualmente se conoce como mercadeo 3.0 (Kotler et al., 2018). En el transcurso de los años, se evidencian variedad de conceptos en los que diferentes autores definen el mercadeo relacional, donde se exhiben diferentes pensamientos del como poder mantener e intensificar las relaciones con los clientes (Chan-Tien et al., 2019). El marketing ayuda a que haya relaciones con los clientes y que estas sean mucho más duraderas a lo largo del tiempo. También se centra en la gestión de la relación entre la empresa y sus clientes, claro está que, para facilitar el éxito de esa gestión, se debe considerar que otros agentes del mercado pueden estar implicados en la misma, como lo pueden ser proveedores, distribuidores, socios e instituciones financieras (Barroso \& Martín, 1999, pp. 34-35).

\subsection{Marketing relacional: mirada desde diferentes autores}

A lo largo de la historia se han expresado diferentes definiciones frente al marketing relacional, plasmando su perspectiva en cuanto al tema. Grönroos (1996) expresa el marketing de relaciones como la identificación, el establecimiento, el mantenimiento y la mejora de las relaciones con los clientes y otras partes interesadas, esto con el fin de generar un benéfico común. En cuanto a Morgan y Hunt (1994) lo definen como todas las actividades de marketing dirigidas a establecer, desarrollar y mantener intercambios relacionales, que resulten de manera exitosa. En términos del contexto 
educativo, el marketing relacional abarca estrategias diseñadas para atraer, establecer y consolidar la relación con las partes interesadas, como son los estudiantes, padres, grupos de referencia y agencias gubernamentales de gran relevancia, con énfasis en la retención de estudiantes y en la búsqueda de nuevos prospectos (Moore \& BowdenEverson, 2012), pero anteriormente Grönroos (1994) lo definiría en la base del relacionamiento se mantiene en la identificación de necesidades para satisfacerlas desde el desarrollo de productos que generen cercanía con los consumidores (Dzimińska et al., 2018), basados en el intercambio y beneficio para quienes intervienen en el proceso organizacional (Liu et al., 2016).

Por otra parte, el marketing de relaciones se define como el intercambio a largo plazo, en donde se puedan establecer y generar relaciones con los clientes (Alet i Vilaginés, 1994) y que para Christopher et al. (1994) ayuda a que se logre una "realineación", y que esto se enfoque en dos puntos, el de captar la atención de los clientes y la retención de los mismos (Popovic, 2006).

\subsection{La gestión educativa y su importancia desde el marketing}

El marketing educativo se ha convertido en el principal motor de cambio del sector de la enseñanza (Llorente, 2019), el cual, es una adaptación del marketing de servicios que le sirve a las instituciones educativas para el diseño de nuevas estrategias, de manera que estas les permitan ampliar la cobertura estudiantil y así satisfacer los diferentes intereses de los miembros de la comunidad académica, planificando, desarrollando y divulgando los programas académicos (Ospina-Díaz \& Sanabria-Rangel, 2010). Además, las estrategias del marketing educativo son crear y facilitar a los clientes-consumidores, en este caso los estudiantes, el poder interactuar en situaciones de aprendizaje participativo dentro y fuera de contextos educativos, con la convicción de que el aprendizaje es un proceso vivencial, fruto de la reflexión, la discusión, el análisis, la interacción (Khoshtaria et al., 2020) y retroalimentación grupal (Naranjo, 2011). Esto mismo, es el proceso de investigación de necesidades sociales, tendientes a desarrollar y a la elaboración de nuevos proyectos educativos que cumplan con las necesidades de las mismas (Hashim et al., 2020), asimismo generando un crecimiento del individuo, esto a través del desarrollo de los servicios educativos (Solís, 2004).

El marketing se ha instituido como una herramienta de innovación, para que las universidades logren su presencia y consolidación en el mercado educativo (Mogaji \& Yoon 2019). Es importante resaltar que el uso del marketing solamente tenía lugar en organizaciones que se dedicaban a la comercialización de productos, sin embargo, el avance de la disciplina ha ganado mayor credibilidad, viéndose este avance principalmente en el campo del comportamiento del consumidor (Peralta-Silva \& Linares-Cazola, 2013). Con los cambios del entorno y el progreso en el conocimiento, el concepto y las aplicaciones del marketing han ido evolucionado (Kureshi \& Thomas, 2020), ya que esta era entendida inicialmente como una disciplina que estudiaba las actividades de distribución desde el productor al consumidor, actualmente el marketing se considera que forma parte de las ciencias humanas, cuyo ámbito no se limita únicamente a la actividad económica, sino también a otras actividades en las que se desempeñan las personas. Esta evolución se pone de manifiesto en la reciente definición de la AMA, en la que se aprecia con más precisión las características esenciales del marketing como una función organizacional, como un conjunto de actividades para crear y proporcionar valor, de 
modo que aporte beneficios para todas las personas e instituciones implicadas en sus actividades (Ponce, 2005). De la misma manera Córdoba (2009) indica que el marketing de relaciones no solo tiene como fin la fidelización en los clientes y el generar mayores ingresos, sino también que las relaciones con los consumidores, perduren a largo plazo, en donde se encuentren beneficios mutuos.

\section{Método}

El presente trabajo se desarrolló con una metodología donde se implementó el método de revisión sistemática de literatura con el cual se permite tener una mayor exactitud y sintetización de la información obtenida (Beltrán, 2005), con el fin de dar respuesta al objetivo por medio de la pregunta de investigación planteada (Tranfield et al., 2003), con la utilización de las bases de datos Dialnet, Scopus, Redalyc, SciELO y google Schoolar, cuyas palabras claves de búsqueda fueron "marketing relacional" y “m marketing en educación”. Los criterios de inclusión para este articulo fueron libros y artículos publicados en Iberoamérica y Latinoamérica, de la misma manera que los temas principales estuvieran enfocados en el marketing de los servicios educativos. El único criterio de exclusión se dio en los trabajos de grados, además no se consideró el tiempo o fecha para la resolución del trabajo, que para el resultado final de la búsqueda deja 61 artículos analizados a nivel nacional e internacional.

La investigación presentada es de tipo exploratorio, debido a que se entra a analizar un tema donde no se encuentran muchas respuestas y no se puede dar a ciencia cierta una respuesta concreta (Sabino, 2014). Los análisis realizados identifican los diferentes cambios generacionales entre los años 2000 hasta 2018 del concepto de marketing relacional, lo cual se visualiza en la tabla 1, donde seguidamente en la tabla 2 y 3 se plantean los diferentes modelos creados y aplicados en Colombia y España. La muestra a evaluar es obtenida del ranking QS, donde se toman las diez mejores universidades de Colombia y España, donde se contemplan las diferentes estrategias de marketing relacional que estas utilizan para llegar a sus grupos de interés.

\section{Resultados}

Los resultados fueron clasificados de la siguiente manera para dar respuesta al objetivo planteado en esta investigación, inicialmente se presentará en la tabla 1 la descripción de la evolución del mercadeo relacional desde el año 2003 al 2018, posterior se mostrará la tabla 2 que contiene los modelos de mercadeo relacional construidos y aplicados en Colombia, así mismo en la tabla 3 se desglosan los modelos de marketing relacional analizados en España, de otro lado en la tabla 4 se realiza un comparativo entre las variables de los modelos de marketing relacional más utilizadas por las universidades de los países anteriormente mencionados, en consiguiente en la tabla 5 se identifica el top 10 de las universidades de ambos países y finalmente se concluyen dos gráficas que sintetizan la importancia de la comunicación digital y la clasificación de los diferentes grupos de interés. 
Tabla 1. Evolución del concepto de marketing relacional

\begin{tabular}{|l|l|}
\hline \multicolumn{1}{|c|}{ Concepto } & \multicolumn{1}{c|}{ Bibliografía } \\
\hline $\begin{array}{l}\text { Se comprende el relacionamiento como el intercambio } \\
\text { repetitivo, donde existe un beneficio para quienes in- } \\
\text { tervienen en la transacción }\end{array}$ & (San Martín, 2003) \\
\hline $\begin{array}{l}\text { Crear y comunicar procesos que generan valor a los } \\
\text { individuos, con el objetivo de perdurar en relaciones } \\
\text { que beneficien la empresa y los consumidores }\end{array}$ & (AMA, 2005) \\
\hline $\begin{array}{l}\text { "El marketing relacional es el proceso de administrar } \\
\text { las relaciones de la empresa de una manera rentable" }\end{array}$ & $\begin{array}{l}\text { (López-Pinto Ruiz, Mas-Machuca, \& } \\
\text { Viscarri-Colomer, 2008, p. 363) }\end{array}$ \\
\hline $\begin{array}{l}\text { Proceso donde se construyen, gestionan y perduran re- } \\
\text { laciones con los grupos de interés }\end{array}$ & (Wakabayashi, 2010) \\
\hline $\begin{array}{l}\text { Desarrollo de relacionamiento duradero con los clientes } \\
\text { para encontrar lealtad y rentabilidad organizacional }\end{array}$ & (Gummesson, 2011) \\
\hline $\begin{array}{l}\text { "Busca gestar la relación de intercambio que se genera } \\
\text { y mantenerla en el tiempo o romperla en determinado } \\
\text { momento" }\end{array}$ & (Mejía-Galeano, 2014, p. 156) \\
\hline $\begin{array}{l}\text { "El marketing relacional podría ser una estrategia que } \\
\text { aporte al fortalecimiento de vínculos entre las Institu- } \\
\text { ciones de Educación Superior IES y sus stakeholders } \\
\text { dentro del proceso de internacionalización" }\end{array}$ & $\begin{array}{l}\text { (Palacio de la Cruz \& Rondón , 2018, } \\
\text { pp. 1-2) }\end{array}$ \\
\hline
\end{tabular}

Fuente: Elaboración propia.

En cuanto a la tabla 1, se puede observar la evolución del concepto de marketing relacional en los últimos 15 años, definido por variedad de autores que han tenido gran importancia en cuanto al impacto, no solo del marketing relacional, sino también del marketing en general (Gómez \& Uribe, 2016). Se puede concluir de la tabla 1 que el concepto no ha cambiado mucho, pero que ha sido adaptado a las diferentes etapas de la evolución del marketing, esto de la misma manera ayudando al sector empresarial y educativo a entender cuál es el camino a seguir para conseguir aquello que desean alcanzar.

Con la identificación de literatura encontrada por diferentes revistas de Colombia y España se puede llegar a afirmar que el país en donde se han realizado más estudios sobre modelos de marketing relacional en educación, ha sido España. Se denota que dicho país posee grandes institutos donde sobresale la investigación en el tema del marketing, en las cuales como resultado se generan modelos que ayudan a medir variables como la imagen, calidad, confianza, satisfacción, identificación y lealtad, aportando en gran medida a la gestión organizacional que beneficia el bienestar de quienes integran la comunidad académica y generando acciones a partir de temas como la motivación y la transformación de individuos que cada día sean más felices en las organizaciones para que construyan sociedad (Foncubierta-Rodríguez \& SánchezMontero 2019). En la siguiente tabla se visualizarán algunos modelos aplicados en Colombia en cuanto al marketing relacional. 


\section{Tabla 2. Modelos aplicados y desarrollados en Colombia en marketing relacional}

\begin{tabular}{|c|c|}
\hline Autor & Objetivo del modelo \\
\hline (Duque, 2005) & $\begin{array}{l}\text { Entender los conceptos de servicio y calidad del servicio, para com- } \\
\text { prender el objeto y las características de los modelos de medición y de } \\
\text { esta forma identificar qué modelo de medición se puede aplicar. }\end{array}$ \\
\hline (Duque, 2009) & $\begin{array}{l}\text { Socializar las diferentes perspectivas basadas en stakeholders para } \\
\text { implementar una estrategia centrada en reforzar el vínculo entre los } \\
\text { clientes y la organización. (Universidad y sus clientes). }\end{array}$ \\
\hline $\begin{array}{l}\text { (Ospina-Díaz \& Sana- } \\
\text { bria-Rangel, 2010) }\end{array}$ & $\begin{array}{l}\text { Proponer el modelo MIGME en el cual se ayudará a la mejora de las } \\
\text { instituciones de manera que se ajuste mejor a las necesidades de la so- } \\
\text { ciedad y del sector productivo. }\end{array}$ \\
\hline $\begin{array}{l}\text { (Vergara-Schmalbach, } \\
\text { Garavito-Díaz, Gue- } \\
\text { rra-Mercado, \& Pos- } \\
\text { so-Quintana, 2015) }\end{array}$ & $\begin{array}{l}\text { Encontrar una herramienta válida, que se amolde a la situación actual } \\
\text { y que con su aplicación permita identificar lo más relevante, que afecta } \\
\text { directamente la satisfacción de los estudiantes. }\end{array}$ \\
\hline $\begin{array}{l}\text { (Nader, Peña-Bernate, } \\
\text { \& Sánchez-Santa-Bár- } \\
\text { bara, 2014) }\end{array}$ & $\begin{array}{l}\text { Determinar si la percepción del clima social, la descripción del trabajo } \\
\text { (significación, responsabilidad y conocimiento de resultados) y la ex- } \\
\text { periencia de fluidez permiten predecir la satisfacción y el bienestar en } \\
\text { el trabajo. }\end{array}$ \\
\hline $\begin{array}{l}\text { (Amaya-Guio \& Carre- } \\
\text { ra-Siabato, 2014) }\end{array}$ & $\begin{array}{l}\text { Aplicar el método MUSA a los resultados de las encuestas del estudio } \\
\text { para monitorear la satisfacción y en pro de diagnosticar el nivel de } \\
\text { satisfacción actual de los estudiantes de pregrado e identificar la im- } \\
\text { portancia y el desempeño de cada uno de los aspectos evaluados por la } \\
\text { institución. }\end{array}$ \\
\hline (Castro, 2014) & $\begin{array}{l}\text { Fortalecer las relaciones de la Universidad Nacional de Colombia, sede } \\
\text { Bogotá y sus egresados, mediante la generación de una propuesta de } \\
\text { valor que permita un beneficio mutuo. }\end{array}$ \\
\hline (Guerrero, 2015) & $\begin{array}{l}\text { Dirigirse al programa educativo y la denominada mezcla de marketing } \\
\text { educativo: satisfactor, intercambio, facilitación y comunicación, que } \\
\text { modifica la mezcla en el mercado tradicional: producto, precio, distri- } \\
\text { bución y promoción. }\end{array}$ \\
\hline
\end{tabular}

Fuente: Elaboración propia.

La tabla 2 permite observar los diferentes modelos aplicados y desarrollados en Colombia a través de la historia; se denota que en este país los modelos educación siguen con un método tradicional, donde muchas universidades no se han dado cuenta que para poder hacer que sus servicios sean aún más conocidos y que los estudiantes y los demás grupos de interés les sean más constantes, deben cambiar su mentalidad o estrategia que generen y busquen el incentivo hacia dicho tema, es decir, implementar nuevas alternativas, tales como nuevos modelos que les ayude a fortalecer relaciones e innovar en sus estrategias, haciéndolas un más aptas para aquello que buscan los distintos grupos de interés a los que se dirigen.

Toda institución de educación superior debe de ser consciente que en la época actual hay una mayor oferta en los servicios educativos, ya sea presencial y/o virtual y 
es por esto que las universidades deben ser estrategas y empezar a pensar en que las decisiones que tomen van a ser las mejores para un futuro (Arrubla-Zapata, 2013). A continuación, la siguiente tabla mostrará los modelos de marketing relacional aplicados en España.

Tabla 3. Modelos aplicados y desarrollados de marketing relacional en España

\begin{tabular}{|l|l|}
\multicolumn{1}{|c|}{ Autor } & \multicolumn{1}{|c|}{ Objetivo del modelo } \\
\hline $\begin{array}{l}\text { (Rueda Osuna \& Rosa } \\
\text { Díaz, 2010) }\end{array}$ & $\begin{array}{l}\text { Desarrollar un sistema social y económico, que juega con el doble } \\
\text { rol de input y output: interactuando entre sí, ejerciendo influen- } \\
\text { cias y generando influencia en los demás. }\end{array}$ \\
\hline $\begin{array}{l}\text { (De la Fuente Mella, Mar- } \\
\text { zo, Navarro, Riquelme, \& }\end{array}$ & $\begin{array}{l}\text { Permitir a la Facultad de Ingeniería de la Universidad de Talca poder } \\
\text { evaluar la influencia de estas variables propuestas en el constructor } \\
\text { de satisfacción con el personal e indirectamente con la satisfacción } \\
\text { general experimentada por los alumnos con los servicios recibidos. }\end{array}$ \\
\hline $\begin{array}{l}\text { (Schlesinger, Taulet, Boni- } \\
\text { llo, \& Fernández, 2014) }\end{array}$ & $\begin{array}{l}\text { Conceptualizar y medir las variables relativas al comportamiento y } \\
\text { percepción de los egresados. De igual manera en la calidad, lealtad } \\
\text { e identificación del mismo con la Universidad. }\end{array}$ \\
\hline $\begin{array}{l}\text { (Larentis, Antonello, \& } \\
\text { Slongo, 2018) }\end{array}$ & $\begin{array}{l}\text { Desarrollar una cultura interorganizacional, basada en elementos } \\
\text { iniciales como: significados y símbolos compartidos, apertura a } \\
\text { nuevas ideas, frecuencia y calidad de las interacciones, entre otros. } \\
\text { Está influenciado por las relaciones interorganizacionales, a través } \\
\text { de elementos intermedios como confianza relacionada con la in- } \\
\text { formación, las acciones y las personas, el compromiso con las ac- } \\
\text { ciones, los resultados y las personas, los procesos de aprendizaje y } \\
\text { los resultados, que podrán contribuir más tarde con los resultados. }\end{array}$ \\
\hline
\end{tabular}

Fuente: Elaboración propia.

En la tabla 3 se puede observar que aparecen cinco modelos los cuales en materia de investigación son los más relevantes a nivel de España, debido a que son los más utilizados e inclusive desarrollados por las mejores universidades de dicho país, en donde se puede encontrar a la universidad de Valencia y la de Sevilla, siendo estas dos las pioneras en cuanto al tema del marketing. Se puede entender también con dicha tabla, que, en comparación a Colombia, España lleva una cierta ventaja en cuanto a la investigación sobre respectivo tema, debido a la potencia investigativa que este tiene sobre la nación colombiana. Ahora bien, es de entender que la mentalidad española respecto al tema de adaptación a las diferentes etapas de los cambios frente al relacionamiento con los consumidores-clientes, es mucho más estricta y eficaz a la hora de poder asimilar la situación, y cómo se venía tratando anteriormente, para Colombia, se hace un poco más difícil conseguir esta adaptación.

La tabla 4, evidencia las variables más repetidas en cuanto a los modelos anteriormente descritos. 


\section{Tabla 4. Comparación de los diferentes modelos entre Colombia y España enfocados en sus variables más repetitivas}

\begin{tabular}{|l|l|l|}
\multicolumn{1}{|c|}{ Modelos } & \multicolumn{1}{c|}{$\begin{array}{c}\text { Variables más } \\
\text { repetitivas }\end{array}$} \\
\hline $\begin{array}{l}\text { Colombia: Diferentes modelos } \\
\text { basados en los stakeholders } \\
\text { España: Modelo de la Identifi- } \\
\text { cación Egresado-Universidad }\end{array}$ & Creación de valor & $\begin{array}{l}\text { (Duque, 2009) } \\
\text { (Schlesinger, Taulet, Bonillo, \& } \\
\text { Fernández, 2014) }\end{array}$ \\
\hline $\begin{array}{l}\text { Colombia: Modelo de sendero } \\
\text { España: Modelo teórico de la } \\
\text { relación egresado-universidad }\end{array}$ & Satisfacción & $\begin{array}{l}\text { (Nader, Peña-Bernate, \& Sán- } \\
\text { chez-Santa-Bárbara, 2014) } \\
\text { (Schlesinger, Taulet, Bonillo, \& } \\
\text { Fernández, 2014) }\end{array}$ \\
\hline $\begin{array}{l}\text { Colombia: La escuela nórdica y } \\
\text { la escuela norteamericana } \\
\text { España: Modelo de ecuaciones } \\
\text { estructurales }\end{array}$ & Confianza & $\begin{array}{l}\text { (Duque, 2005) } \\
\text { (Schlesinger, Cervera, \& Pérez-Ca- } \\
\text { bañero, 2017) }\end{array}$ \\
\hline
\end{tabular}

Fuente: elaboración propia.

En la tabla 4 se identifican los diferentes modelos estudiados entre Colombia y España y de la misma manera se hace una comparación de las variables que más analizan estos países y también en las semejanzas que estos tienen.

En el primer ítem que contiene la tabla 4 se evidencia que los modelos tanto en Colombia como en España se repite la variable creación de valor, en donde cada modelo tiene un enfoque específico para esta, es decir, tienen una perspectiva diferente. En el modelo colombiano esta variable se enfoca en como la universidad crea valor en cada uno de los grupos de interés, no solo fijándose en los estudiantes, sino en cada uno de los grupos que integran la universidad, sean estos directivos, secretarios, profesorado, entre otros (Duque, 2009). En el modelo español se intenta mirar que tan enfocado está el alumno con la universidad y así mismo darse cuenta si estos están realizando de una manera adecuada su trabajo o no (Schlesinger et al., 2014).

Analizando ahora el segundo ítem que contiene la tabla 4 se denota uno de los pilares principales que tiene una universidad, el cual es la identificación del nivel de satisfacción que poseen sus estudiantes con las universidades. Se puede observar que la variable satisfacción en España, busca medirse en los egresados, intentado saber que tan conformes se encuentran con la institución en la que realizaron e inclusive aun realizan sus estudios y de igual forma identificar cuales están siendo sus falencias y poder mejorarlas (Schlesinger et al., 2014). En Colombia dicha variable busca medir la satisfacción de los estudiantes, identificando si estos se encuentran conformes con los docentes con y demás administrativos, donde se busca que haya un bienestar de trabajo equilibrado. (Nader et al., 2014)

En cuanto al último ítem de la tabla con los dos modelos restantes, se puede observar la variable confianza, en donde se identifica que, en España esta busca medirse respecto a la imagen que tienen los estudiantes de la universidad y si estos confían en que la institución realmente está haciendo su labor de la mejor manera 
(Schlesinger et al., 2015). Ahora bien, en cuanto a Colombia, los estudiantes no miran esta confianza en el sector interno, sino en el externo, identificando que tan confiable es la universidad en el sector, siendo esto una manera de percibir lo confiable que es la universidad para los agentes externos a la institución, aquellos que no hacen parte de la misma (Duque, 2005).

España como pionera es uno de los principales países en investigación y propuestas en cuanto al tema de marketing, este da en evidencia a una de las mejores universidades con más investigaciones y trabajos al respecto y es la universidad de Valencia, siendo una de las más prestigiosas de este país. No solamente dicha universidad se encuentra entre las investigadoras y principales exponentes del tema del marketing, pues también se puede encontrar cantidad de resultados expuestos por variedad de autores en la universidad de Sevilla.

Comparando a Colombia con España, se observa que las investigaciones del país latinoamericano, vienen siendo un pilar importante en cuanto al tema de marketing, pero que a nivel de España aún sus investigaciones se quedan cortas, no en el tema de la calidad de estas, sino en la cantidad de investigaciones que se generan, sin embargo, en Colombia se pueden encontrar universidades que le apuntan de gran manera al tema de la investigación del marketing, específicamente en el marketing relacional.

Teniendo en cuenta la revisión de literatura se evidencian algunas estrategias de marketing relacional tanto en universidades de Colombia como de España y que se enfocan en las variables más repetidas manifestadas en la tabla 4 que son creación de valor, confianza y satisfacción. En las de creación de valor tienen flexibilidad en los procesos tanto académicos como administrativos para que los estudiantes puedan ver las asignaturas del plan de estudio y pagar por medio de financiaciones y alianzas con entidades. En cuanto a la confianza construyen estrategias enmarcadas en la coherencia entre lo que prometen las universidades y lo que le brindan a quienes integran la comunidad académica. Las estrategias más representativas en satisfacción para las universidades están en el mejoramiento constante de la infraestructura y espacios que les brinden experiencias significativas a los individuos, al mismo tiempo en la calidad de los docentes y de los procesos académicos que implementan las universidades a nivel nacional e internacional.

\subsection{Estrategias de las universidades top según el ranking $Q S$}

Según el ranking QS, que es el encargado de denominar el listado de las mejores universidades a nivel mundial, en la tabla 5 se evidencia el top 10 de las universidades mejor calificadas de los países de Colombia y España. (QS Quacquarelli Symonds Limited)

\section{Tabla 5. Top 10 de las universidades de Colombia y España}

\begin{tabular}{|c|c|c|}
\hline País & Logo & Universidad \\
\hline 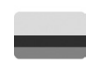 & 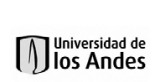 & Universidad de los Andes \\
\hline 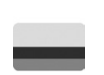 & 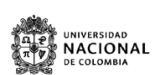 & Universidad Nacional de Colombia \\
\hline
\end{tabular}




\begin{tabular}{|c|c|c|}
\hline País & Logo & Universidad \\
\hline & $\begin{array}{l}\text { U. UNIVERIDAD } \\
\text { DEANTIOQUIA }\end{array}$ & Universidad de Antioquia \\
\hline & 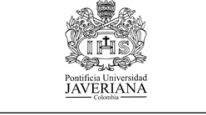 & Pontificia Universidad Javeriana \\
\hline & 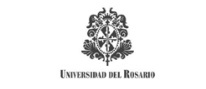 & Universidad del Rosario \\
\hline 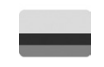 & SIJ Universidad del Valle & Universidad del Valle \\
\hline 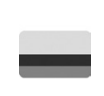 & 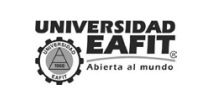 & Universidad EAFIT \\
\hline 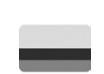 & 9. Lumasioddce & Universidad de la Sabana \\
\hline 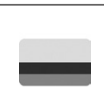 & 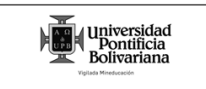 & Universidad Pontificia Bolivariana \\
\hline 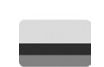 & 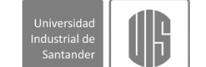 & Universidad Industrial de Santander \\
\hline
\end{tabular}

\begin{tabular}{|c|c|c|}
\hline País & Logo & Universidad \\
\hline 意 & 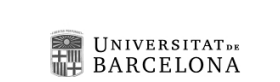 & Universidad de Barcelona \\
\hline 就 & $\underset{\text { Universitat Autònoma }}{\mathbf{A B}}$ & Universidad Autónoma de Barcelona \\
\hline 港 & UÁM'M & Universidad Autónoma de Madrid \\
\hline : & 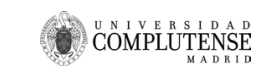 & Universidad Complutense de Madrid \\
\hline 趡 & $\begin{array}{l}\text { 1. Universidad } \\
\text { de Navarra }\end{array}$ & Universidad de Navarra \\
\hline 葓 & 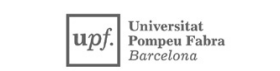 & Universidad Pompeu Fabra \\
\hline 索 & 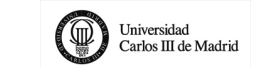 & Universidad Carlos III de Madrid \\
\hline 唩 & 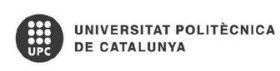 & Universidad Politécnica de Catalunya \\
\hline 索 & UNIVERSITY & IE University \\
\hline 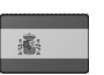 & $\begin{array}{l}\text { UNIVESITAT } \\
\text { POIIIECNICA } \\
\text { DE VIENCIA }\end{array}$ & Universidad Politécnica de Valencia \\
\hline
\end{tabular}

Fuente: Elaboración propia basada en el ranking QS. 
En la tabla 5 se observa que España en su top 10 de universidades tiene a dos instituciones que son ampliamente reconocidas por sus investigaciones en el tema de marketing relacional a nivel educativo, las cuales son la universidad de Navarra y el IE University, en las cuales se pueden encontrar algunos documentos por parte de la universidad de Navarra en referencia al marketing, pero que las universidades que más investigaciones realizan sobre marketing relacional no aparecen en este top. De la misma manera, en cuanto a Colombia en su top 10 aparecen tres universidades muy bien posicionadas, donde las investigaciones acerca del tema son de gran relevancia. Entre estas instituciones se encuentran la universidad Nacional de Colombia, siendo esta la más relevante y aportadora al marketing, haciendo grandes aportes no solo a su entidad, sino también a la comunidad educativa a nivel nacional. Además, se encuentran la universidad de los Andes y la universidad EAFIT, las cuales también hacen aportes investigativos al marketing.

Con el fin de sintetizar las diferentes estrategias que a nivel comunicacional implementan las universidades exploradas, se presenta en el gráfico 1 el esquema de comunicación por redes sociales y en el gráfico 2 los grupos de interés con los que más se relacionan, este esquema se logra a partir de técnica de observación con fichas de relación por universidades y por la usabilidad de las herramientas digitales. Se pueden denotar las diferentes estrategias de comunicación implementadas por las universidades mencionadas en la tabla 5 , donde se evidencian sus redes sociales y el impacto que tienen estas en el ámbito educativo para un mejor relacionamiento de la institución con sus grupos de interés como se demuestra en el gráfico 2, observando, así como las universidades tienen un enfoque de mirada diferente para los distintos grupos a los que desean dirigirse.

\section{Gráfico 1. Redes sociales utilizadas por las universidades de Colombia y España}

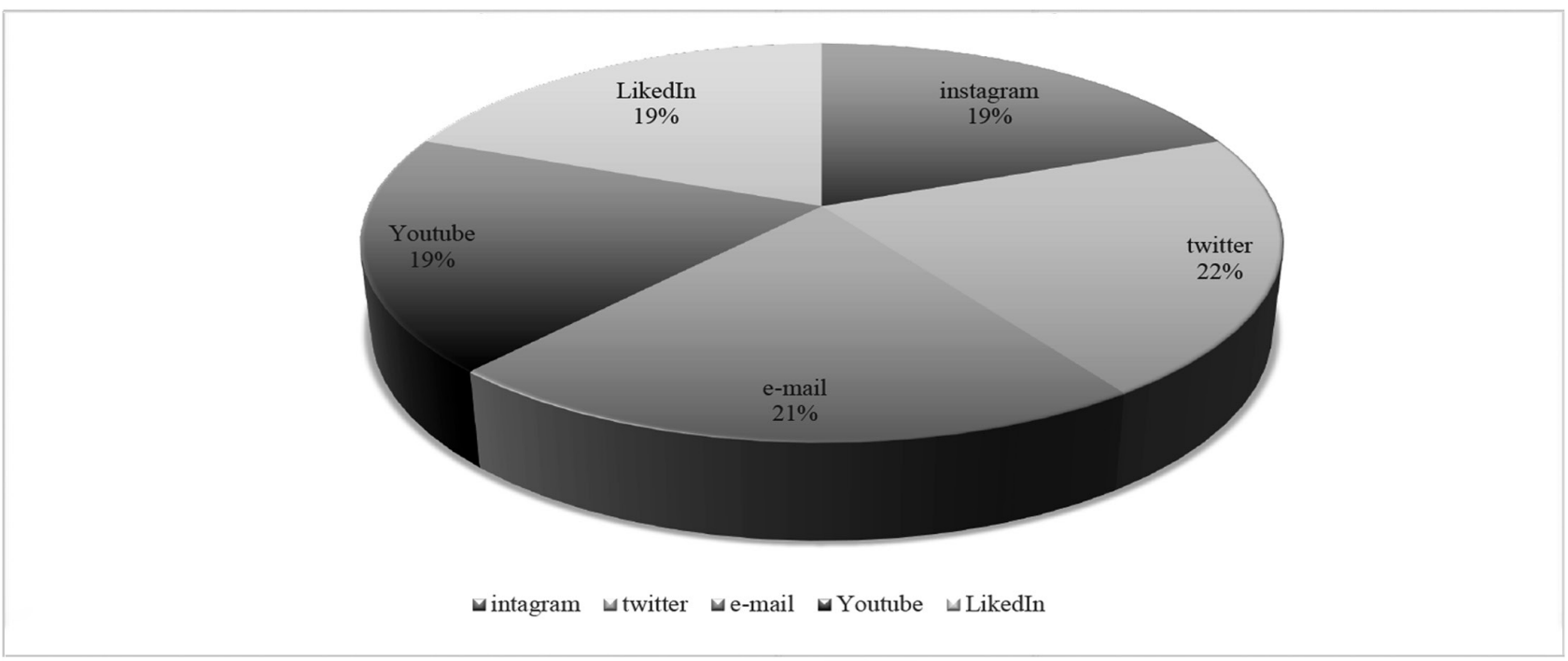




\section{Gráfico 2. Grupos de interés a los que se dirigen las universidades de Colombia y España}

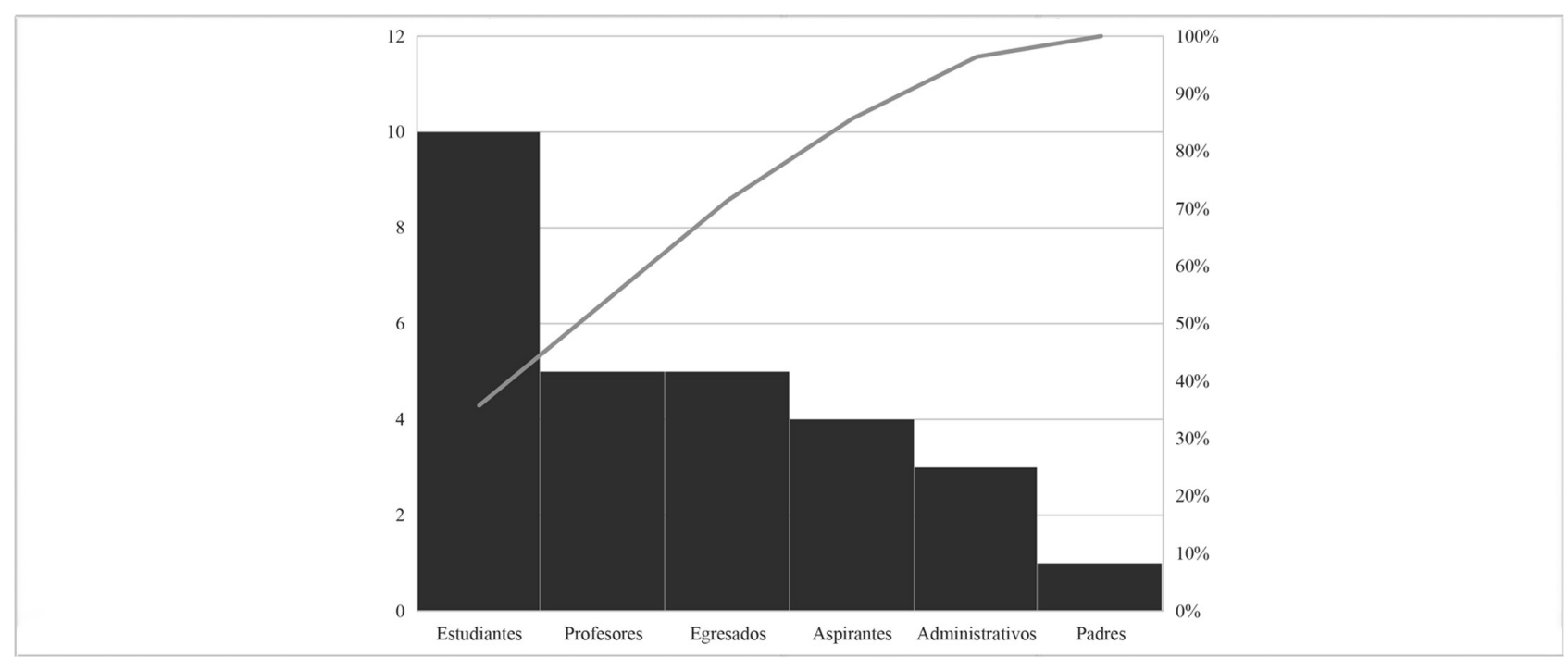

Fuente: Elaboración propia

Es importante recalcar que las universidades en España en cuanto a estrategias que realizan para dar facilidades de estudio a sus grupos de interés, en mayor ámbito a los estudiantes, prestan servicios de vivienda en donde las personas foráneas a la ciudad o el país puedan acceder de una manera fácil y conveniente, además como servicios financieros apoyan mediante becas, donaciones e intercambios a diferentes universidades alrededor del mundo. En cuanto a las becas y donaciones, estas también pueden hacerse por parte de los estudiantes y profesorado, dando ciertas donaciones, sean económicas o de temas de estudio como libros, en donde faciliten la entrada de nuevas personas a la institución educativa.

\section{Conclusiones y discusiones}

Se concluye que el marketing relacional ha venido ganando importancia en los esquemas de trabajo para la gestión administrativa de las universidades, se han involucrado diferentes variables que le dan mayor fortaleza a este concepto haciéndolo más atractivo para ser aplicado en los diferentes grupos de interés con los que se comunica la universidad, sin embargo se encuentra que aunque el concepto es importante y requiere mayor atención por los directivos de las universidades, poco ha sido utilizado en los modelos de gestión y son escasas las estrategias que son aplicadas para generar valor.

Así mismo el marketing relacional, cuando se enfoca al ámbito educativo ha traído resultados satisfactorios, no solo para las instituciones que realizan investigaciones, sino que también se aporta a la gestión administrativa de la educación en general, con la construcción de diferentes modelos que implementan estrategias que pueden ser utilizados como guía para lograr mejores beneficios en indicadores de satisfacción y lealtad de la comunidad a la universidad. Con la construcción de modelos se evidencia que tanto en España como el Colombia su enfoque lo hacen a través de tres variables que son creación de valor, satisfacción y la confianza.

Se hace necesario comprender que si bien es cierto se encuentran algunas estrategias enfocadas al marketing relacional que generan valor en las universidades, se debe 
avanzar en la incorporación de estrategias que involucren a los diferentes públicos y que los hagan partícipes de los objetivos mercadológicos planteados que mejoren la visibilidad y rentabilidad de la gestión administrativa, así mismo se evidencian unos esquemas que van centrados en la comercialización de los servicios, más no en la construcción de satisfacción para generar lealtad.

Ahora bien, al analizar los comparativos entre los países se encuentra que España es generador de nuevo conocimiento, en el desarrollo de investigaciones acerca del marketing relacional, han generado varios modelos que las universidades pueden aplicar para generar nuevas estrategias, e incluso para medir variables a las cuales las instituciones desean llegar y medir, dando de la misma manera instrumentos que avalen las respuestas dadas. España también, en este tema de marketing es fuerte, puesto que cuenta con grandes universidades en las cuales las investigaciones acerca del marketing relacional en educación han logrado grandes avances, en donde una de estas universidades, es la universidad de Valencia, que día a dice crece más en este tema, con respecto a Colombia, también se pueden encontrar gran variedad de apartados y documentos que se realizan por universidades reconocidas y bien posicionadas a nivel de Latinoamérica, generando modelos e instrumentos que ayudan a la generación de más conocimiento respecto al tema, sirviendo estos no solo para las instituciones educativas, sino que sirven también para el sector empresarial en cuanto al sector interno de las organizaciones. En este tema de marketing relacional se pueden encontrar universidades como la Universidad Nacional, la EAFIT, Universidad de Antioquia y la Universidad de los Andes, como las más importante en cuanto a estas investigaciones.

Debido a la necesidad de seguir explorando en el tema de marketing en educación, se considera oportuno continuar indagando en la importancia de las estrategias de relacionamiento y comunicación en la gestión educativa a partir de los diferentes grupos de interés con los que interactúan las instituciones, al mismo tiempo comparar las acciones que se hacen en Colombia con otros países diferentes a España para identificar cuales estrategias se pueden contextualizar en el entorno educativo colombiano.

El tema del marketing relación ha venido tomando fuerza a través de la historia, donde ya no sólo se enfocan en un público, sino que evalúan todos sus grupos de interés para que con esto puedan identificar las mejores estrategias que deberán implementar para generar un mejor valor agregado, y desde una mirada educativa, las instituciones de educación superior deben de mantener una constante en evolución en aspecto puntuales como su marca, atención y redes sociales, las cuales son las variables más visibles que tiene la comunidad interna como externa para conocer de la universidad.

\subsection{Apoyos y soporte financiero de la investigación}

Entidad: Universidad de San Buenaventura seccional Medellín

País:Colombia

Ciudad: Medellín

Proyecto Mercadeo relacional en educación superior 2019

\section{Referencias}

Alet i Vilaginés, J. (1994). Marketing relacional: cómo obtener clientes leales y rentables. Gestión 2000. AMA. (2005). American Marketing Asociation.

Amaya-Guio, C.A., \& Carrera-Siabato, N. (2014). Utilización de la metodología MUSA y su apli- 
cación en el estudio para monitorear periódicamente la satisfacción de estudiantes de la Universidad de los Andes (ESAT). Revista de Ingeniería, (40), 20-26. https://bit.ly/33C08Ou

Arnett, D.B., German, S.D., \& Hunt, S.D. (2003). The identity salience model of relationship marketing success: The case of nonprofit marketing. Journal of Marketing, 67(2), 89-105. doi. org/10.1509/jmkg.67.2.89.18614

Arrubla-Zapata, J.P. (2013). Marketing en universidades. Análisis de factores críticos de competitividad. Escenarios: empresa y territorio, 2(2), 207-226.

Barroso, C., \& Martín, E. (1999). Marketing relacional. ESIC.

Beltrán, O.A. (2005). Revisiones sistemáticas de la literatura. Revista colombiana de gastroenterología, 20(1), 60-69.

Bryce, H.J. (2007). The public's trust in nonprofit organizations: The role of relationship marketing and management. California Management Review, 49(4), 112-131. doi.org/10.2307/41166408

Castro, G.D. (2014). Modelo de marketing relacional para el fortalecimiento de las relaciones Universidad-Egresado. Caso Universidad Nacional de Colombia, sede Bogotá. (Doctoral dissertation, Universidad Nacional de Colombia).

Ceballos-Lozano, A., Arévalo-Fajardo, B., \& Giraldo-Agudelo, J. (2012). Plan de Marketing para incrementar el número de estudiantes en el Centro Educativo la Sabiduría de la Ciudad de Barranquilla. Escenarios, 10(1), 29-39.

Córdoba-López, J.F. (2009). Del marketing transaccional al marketing relacional. Entramado, 5(1), 6-17. https://bit.ly/2XNnLjN

Cownie, F. (2019). What drives students' affective commitment towards their university?. Journal of Further and Higher Education, 43(5), 674-691. doi.org/10.1080/0309877X.2017.1394988

Christopher, M., Payne, A., \& Ballantyne, D. (1994). Marketing relacional: integrando la calidad, el servicio al cliente y el marketing. Ediciones Díaz de Santos.

Chan-Tien, L., Dan-Sheng, W., \& Shun-Fa, H. (2019, June). The Conceptual Framework for Applying Digital Community Marketing and Marketing Practices into Educational Relationship Marketing Model of Private Technical High School in Taiwan. In Proceedings of the 2019 International Conference on Modern Educational Technology (pp. 14-19).

De la Fuente-Mella, H., Marzo-Navarro, M., Riquelme, R., \& Jesús, M. (2010). Análisis de la satisfacción universitaria en la Facultad de Ingeniería de la Universidad de Talca. Revista chilena de ingeniería, 18(3), 350-363. doi.org/10.4067/S0718-33052010000300009

Dzimińska, M., Fijałkowska, J., \& Sułkowski, Ł. (2018). Trust-based quality culture conceptual model for higher education institutions. Sustainability, 10(8), 2599.

doi.org/10.3390/su10082599

Duque-Oliva, E.J. (2005). Revisión del concepto de calidad del servicio y sus modelos de medición. INNOVAR. Revista de Ciencias Administrativas y Sociales, 15(25), 64-80. https://bit.ly/3jvRV3b

Duque-Oliva, E.J. (2009). La gestión de la universidad como elemento básico del sistema universitario: una reflexión desde la perspectiva de los stakeholders. INNOVAR. Revista de Ciencias Administrativas y Sociales, 19, 25-41. https://bit.ly/34Pww0T

Fernández, G., Vázquez, J.M., \& Corredoira, M. D. (2007). La importancia de los stakeholders de la organización: un análisis empírico aplicado a la empleabilidad del alumnado de la universidad española. Investigaciones europeas de dirección y economía de la empresa, 13(2), 13-32. https:// bit.ly/31o0NAv

Foncubierta-Rodríguez, M.J., \& Sánchez-Montero, J.M. (2019). Hacia la felicidad laboral: Atender motivaciones y eliminar temores digitales. Retos. Revista de Ciencias de la Administración y Economía, 9(18), 239-257. doi.org/10.17163/ret.n18.2019.04

Gómez, L., \& Uribe, J. (2016). Marketing relacional: La evolución del concepto. Revista Espacios, 37(25), 11.

Grönroos, C. (1994). From marketing mix to relationship marketing: Towards a paradigm shift in marketing. Asia-Australia Marketing Journal, 2(1), 9-29. doi.org/10.1016/S1320-1646(94)70275-6

Grönroos, C. (1996). Relationship marketing: strategic and tactical implications. Management decision. doi.org/10.1108/00251749610113613 
Guerrero, E. (2015). Mercadeo Educativo ¿Cómo promover la oferta de instituciones y programas? Universidad de los Andes.

Gummesson, E. (2011). Total relationship marketing. Routledge.

Hashim, S., Mohd Yasin, N., \& Ya'kob, S.A. (2020). What constitutes student-university brand relationship? Malaysian students' perspective. Journal of Marketing for Higher Education, 1-23. doi. org/10.1080/08841241.2020.1713278

Hennig-Thurau, T., Langer, M., \& Hansen, U. (2001). Modeling and managing student loyalty: An approach based on the concept of relationship quality. Journal of service research, 3(4), 331-344. doi.org/10.1177/109467050134006

James, C., Crawford, M., \& Oplatka, I. (2019). An affective paradigm for educational leadership theory and practice: connecting affect, actions, power and influence. International Journal of Leadership in Education, 22(5), 617-628. doi.org/10.1080/13603124.2018.1481536

Kotler, P., Kartajaya, H., \& Setiawan, I. (2018). Marketing 4.0: Transforma tu estrategia para atraer al consumidor digital. LID Editorial.

Kureshi, S., \& Thomas, S. (2020). Testing the influence of message framing, donation magnitude, and product category in a cause-related marketing context. Journal of Marketing Communications, 26(3), 268-289. doi.org/10.1080/13527266.2018.1528475

Khoshtaria, T., Datuashvili, D., \& Matin, A. (2020). The impact of brand equity dimensions on university reputation: an empirical study of Georgian higher education. Journal of Marketing for Higher Education, 1-17. doi.org/10.1080/08841241.2020.1725955

Larentis, F., Antonello, C.S., \& Slongo, L.A. (2018). Organizational culture and relationship marketing: an interorganizational perspective. Revista Brasileira de Gestão de Negócios, 20(1), 37-56. doi. org/10.7819/rbgn.v20i1.3688

Llorente, A. C. (2019). Marketing educativo: Captación y fidelización de alumnos. ESIC Editorial.

Liu, S., Hallinger, P., \& Feng, D. (2016). Learning-centered leadership and teacher learning in China: Does trust matter?. Journal of Educational Administration. doi.org/10.1108/JEA-02-2016-0015

Mejía-Galeano, D.Y. (2014). Recompras de programas académicos en educación superior: los factores decisivos desde el marketing. Punto de vista, 5(8). https://bit.ly/3gxu0PX

Moore, D., \& Bowden-Everson, J.L.H. (2012). An appealing connection-the role of relationship marketing in the attraction and retention of students in an australian tertiary context. Canadian Center of Science and Education, 8(14). doi.org /10.5539/ass.v8n14p65

Mogaji, E., \& Yoon, H. (2019). Thematic analysis of marketing messages in UK universities' prospectuses. International Journal of Educational Management, 33(7). doi.org/10.1108/IJEM-05-2018-0149

Morgan, R., \& Hunt, S. (1994). The commitment-trust theory of relationship marketing. Journal of Marketing, 58(3), 20-38. doi.org/10.1177/002224299405800302

Nader, M., Peña-Bernate, S.P., \& Sánchez-Santa-Bárbara, E. (2014). Predicción de la satisfacción y el bienestar en el trabajo: hacia un modelo de organización saludable en Colombia. Estudios Gerenciales, 30(130), 31-39. doi.org/10.1016/j.estger.2014.02.006

Naranjo, C. (2011). Marketing educativo; desarrollo de una estrategia CEM aplicado a la Universidad Nacional de Colombia sede Manizales como base para la fidelización de clientes y complemento a la estrategia de CRM. (Doctoral dissertation, Universidad Nacional de Colombia-Sede Manizales).

Oplatka, I., \& Lapidot, A. (2018). Novice principals' perceptions of their mentoring process in early career stage: the key role of mentor-protégé relations. Journal of Educational Administration and History, 50(3), 204-222. doi.org/10.1080/00220620.2017.1420044

Ospina-Díaz, M.R., \& Sanabria-Rangel, P.E. (2010). Un enfoque de mercadeo de servicios educativos para la gestión de las organizaciones de educación superior en Colombia: el modelo MIGME. Revista Facultad de Ciencias Económicas, 18(2), 107-136.

Palacio de la Cruz, S., \& Rondón, C.A. (2018). Marketing relacional para la internacionalización en instituciones de educación superior. Espacios, 39(23). https://bit.ly/3icFito

Peruta, A., \& Shields, A. B. (2018). Marketing your university on social media: a content analysis 
of Facebook post types and formats. Journal of Marketing for Higher Education, 28(2), 175-191. doi. org/10.1080/08841241.2018.1442896

Ponce, J.M. (2005). Un Nuevo Marketing de las Universidades: Objetivos Formativos y Demandas Sociales. Mier-Terán, JJ and Serrano, C. (Coords.) IV Congreso Internacional de Marketing Público y No Lucrativo. Jerez de la Frontera (Spain): Ayuntamiento de Jerez, electronic edition (CD ROM).

Popovic, D. (2006). Modelling the marketing of high-tech start-ups. Journal of targeting, measurement and analysis for marketing, 14(3), 260-276. doi.org/10.1057/palgrave.jt.5740186

QS Quacquarelli Symonds Limited. (15 de agosto de 2019). Ranking Universidades. https://bit.ly/3a3cVLo

Rueda Osuna, Y., \& Rosa Díaz, I.M. (2010). Un enfoque de marketing educativo holístico en la formación profesional de grado superior. III Jornadas de Investigación e Innovación Docente (2010), 336-350.

López-Pinto Ruiz, B., Mas-Machuca, M., \& Viscarri-Colomer, J. (2008). Los pilares del marketing. Ediciones UPC. https://bit.ly/2XveAUX

Sabino, C. (2014). El proceso de investigación. Episteme.

Sakthivel, P.B., \& Raju, R. (2006). An instrument for measuring engineering education quality from students' perspective. Quality Management Journal, 13(3), 23-34. doi.org/10.1080/10686967.2006.11918559

San Martín, S. (2003). La relación del consumidor con las agencias de viaje (1 ed., Vol. 1). Universidad de Burgos.

Schlesinger, W., Cervera, A., \& Iniesta, M.Á. (2015). Key elements in building relationships in the higher education services context. Journal of Promotion Management, 21(4), 475-491. doi.org/10.1080/10496491.2015.1051403

Schlesinger, W., Cervera, A., \& Pérez-Cabañero, C. (2017). Sticking with your university: the importance of satisfaction, trust, image, and shared values. Studies in Higher Education, 42(12), 2178-2194. doi.org/10.1080/03075079.2015.1136613

Schlesinger, M.W., Cervera, A., Bonillo, M.Á., \& Fernández, R. (2014). Un enfoque de marketing de relaciones a la educación como un servicio: aplicación a la Universidad de Valencia. INNOVAR. Revista de Ciencias Administrativas y Sociales, 24(53), 113-125. doi.org/10.15446/innovar. v24n53.43919

Peralta-Silva, G.O., \& Linares-Cazola, J.G. (2013). Las estrategias de marketing y los niveles de participación de mercado de las universidades 2010. In Crescendo, 4(1), 15-24. https://doi. org/10.21895/incres.2013.v4n1.02

Solís, J.M. (2004). El manejo del marketing en el servicio educativo, en la organización educativa continental. Repositorio de la Universidad Nacional Mayor de San Marcos. Perú.

Tranfield, D., Denyer, D., \& Smart, P. (2003). Towards a methodology for developing evidence-informed management knowledge by means of systematic review. British Journal of Management, 14(3), 207-222. doi.org/10.1111/1467-8551.00375

Trullas, I., Simo, P., Fusalba, O. R., Fito, A., \& Sallan, J. M. (2018). Student-perceived organizational support and perceived employability in the marketing of higher education. Journal of Marketing for Higher Education, 28(2), 266-281. doi.org/10.1080/08841241.2018.1488334

Vergara-Schmalbach, J.C., Garavito-Díaz, D., Guerra-Mercado, L., \& Posso-Quintana, S. (2015). Valoración de las percepciones y satisfacción de los estudiantes sobre el servicio recibido en universidades de Cartagena De Indias-Colombia.

Wakabayashi, J.L. (2010). Research on relational marketing: an analysis on literature content 2007-2008/La investigation sobre el marketing relacional: un analisis de contenido de la literatura 2007-2008. Journal of Economics, Finance and Administrative Science, 15(29), 119-131.

Webster, R., \& Hammond, K. (2008). Does a market orientation strategy exist toward business school students? A view from three levels of academic administrators. Academy of Marketing Studies Journal, 12(2), 19-33. 